\title{
Aging of Reproductive Systems- A Review Article
}

\author{
Heshmat SW Haroun* \\ Department of Anatomy and Embryology, Faculty of Medicine, Cairo University, Egypt
}

Received: 眥 February 27, 2018; Published: 眥 March 06, 2018

*Corresponding author: Heshmat SW Haroun, Department of Anatomy and Embryology, Faculty of Medicine, Cairo University, Egypt Email: heshmatsabet@gmail.l.com/heshmat.haroun@kasralainy.edu.eg.com/heshmat.haroun@scholar.cu.edu.eg

\begin{abstract}
Aging is an irreversible, progressive and complex process that is manifested with macroscopic and microscopic changes in tissues, organs and systems. Human aging starts at the age of 30-40 years. The causes of aging remain unclear but reactive oxygen species and mitochondrial damage are the two acceptable theories. Stimulation of autophagy, caloric restriction and physical exercise are supposed to slow down the aging changes. Thyrotropin releasing hormone and melatonin are considered as antiaging factors. Despite the increase in human life-expectancy, the reproductive organs are the earliest to reveal adverse age-related changes. In male aging there are decreased level of serum testosterone, decline in sperm count and quality, and derangement of androgen target organs such as bone, muscle, and psychosexual function. Older father will likely give rise to an offspring with genetic anomaly, childhood cancer, and or neuropsychiatric disorder. Inflammaging in women is an age-dependent low-grade and chronic inflammation that accelerates reproductive dysfunction in the ovaries, uterine tubes and uterus. Experimental researches have revealed increased fibrosis of ovarian stroma in old female animals. Sex hormones- replacement therapy should be cautiously adopted in aging individuals.
\end{abstract}

Keywords: Aging; Reproductive Organs; Function; Structure

Abbreviations: DHEA: Dehydroepiandrosterone; FSH: Follicle Stimulating Hormone; GH: Growth Hormone; LH: Luteinizing Hormone; MRI: Magnetic Resonance Imaging; PSR: Picrosirius Red; ROS: Reactive Oxygen Species; SHBG: Sex Hormone Binding Globulins; TRH: Thyrotropin Releasing Hormone; TSH: Thyroid Stimulating Hormone

\section{Introduction}

Aging is defined as a gradual, irreversible and complex process in which cells, tissues, organs, and the whole organism progressively deteriorate [1]. Aging is accompanied by major biochemical and biomechanical changes at both macroscopic and microscopic levels that affect tissues and organs as well as cells and their organelles [2]. Although all multicellular organisms undergo structural and functional deterioration with age, senescence is not a uniform process [3]. Aging is mentioned to start at the age of 30-40 years, leading to progressive organ dysfunction which makes keeping homeostasis more difficult. However, body changes previously thought to be due to aging are often proved to be due to pathologies when new features are discovered [4].

The causes of aging are not fully elucidated. Reactive oxygen species (ROS) and mitochondrial theories seem to be the two most evident theories of aging [5]. Aging results in damage of cellular DNA, proteins, membranes, and organelles, as well as accumulation of high molecular weight insoluble aggregates. It has been noted that failure of cellular maintenance and repair is irreversible, although longevity may be modulated through dietary restriction [6]. The somatic mutation theory of aging assumes that accumulation of mutations in the genetic material of somatic cells results in inactivation of genes and decreased cellular function of various organs. When the organ function decreases below a critical threshold, death of the organism occurs [7].

Despite the increase in human longevity, the chronology of the female reproductive cycles and the age onset of menopause have not been changed. The frequency of cancers increases with age due to the increase in somatic mutations and the decline in immune defenses. Drug therapy also must be modified with age, due to age-related changes in pharmacology. Physical exercise and caloric restriction are currently the only measures known to slow the aging process $[8,9]$. 
Damaged proteins and organelles accumulate in aged organs. Autophagy is essential for managing the quality of cellular proteins and organelles to maintain cell function. Inhibition of autophagy decreases the organismal lifespan while stimulation of autophagy can prolong this lifespan [10]. Autophagy is a fundamental autocannibalizing process that preserves cellular homeostasis and, if it is excessive or defective cell function is greatly changed and can result in serious human diseases [11].

\section{Aging of male reproductive system}

The most drastic gender differences in aging are related to the reproductive organs. With the prolongation of life expectancy, both women and men live $1 / 3$ of their life with endocrine deficiencies. In reproductive aging, men experience slow and continuous decline in sex hormones in contrary to women who have rapid decline in sex hormones expressed by the cessation of menses. With aging, there are decreased levels of testosterone, dehydroepiandrosterone (DHEA), estrogens, thyroid stimulating hormone (TSH), growth hormone (GH), and melatonin and increased levels of luteinizing hormone ( $\mathrm{LH}$ ) and follicle stimulating hormone (FSH). Additionally, sex hormone binding globulins (SHBG) are increased with age leading to further lowering of the levels of free active androgens [12]. The decline in serum testosterone level with age has a negative impact on androgen target organs such as bone, muscle, and psychosexual functioning [13]. Nervousness, depression, impaired memory, lack of concentration, insomnia, easy fatigability, hot flushes, periodic sweating, reduction of muscle mass and power, bone ache, and sexual dysfunction are androgen-related changes in aging men [14]. Hormonal replacement therapy may prevent the preventable and delay the inevitable debilitating conditions secondary to endocrine deficiencies [12], but the potential benefits of androgen supplements must be balanced with their potential risks $[13,14]$.

Male aging also causes genetic and epigenetic changes in spermatozoa, inducing adverse effects on sperm quality and count, sex organs and the hypothalamic-pituitary-gonadal axis. Fortunately, recent advanced techniques in assisted reproduction give older men an opportunity to have a child even with poor semen parameters. Older fathers likely give rise to offsprings who show high prevalence of genetic abnormalities, childhood cancers, and neuropsychiatric disorders [15]. Damage accumulated in cells of elderly human males leads to progressive deregulation of the hypothalamic-pituitary-gonadal axis inducing changes in the testis, penis and prostate. Mitochondrial energy is essential for male reproduction and they represent a link between aging and fertility [16].

Oral administration of thyrotropin releasing hormone (TRH) to old male mice revealed rapid correction to more juvenile levels of most typical aging-related hormonal and metabolic measurements. Testicular function was regained in aging mice. There were active formation and proliferation of mature spermatogonia and intensive spermatogenesis. TRH and melatonin are considered as anti-aging factors [17].

\section{Aging of female reproductive system}

In humans, cellular and organic senescence is mentioned to contribute to the age-related decline in reproductive function. Advanced maternal age is associated with loss of fertility and pregnancy complications. Immune cells play a fundamental role in normal reproductive function and pregnancy. Inflammaging is an age-dependent, systemic, low-grade and chronic inflammation induced by the innate immune, parenchymal, and nonparenchymal cells within the organs. It has been stated that cellular senescence and inflammaging accelerate reproductive dysfunction in women particularly in the ovaries (the corpus luteum), oviducts, and uterus [18].

Magnetic resonance imaging (MRI) of the uterus and ovaries has revealed different morphological changes for a given physiological state. During menstruation, the zonal architecture of the uterine corpus is not evident, and the ovarian stroma is shrunken. Around ovulation, the ovarian stroma reaches its maximum size. Functional cysts are often seen in ovaries of reproductive age and they usually spontaneously shrink whereas surface-epithelial inclusion cysts are commonly seen in postmenopausal ovaries. Pregnancy-related morphologies may be seen in the uterus and ovaries mimicking gynecologic tumors and if incorrectly diagnosed lead to unnecessary surgical intervention [19].

Picrosirius Red (PSR), a connective tissue stain specific for collagen I and III fibers, was used in mice to evaluate ovarian fibrosis. PSR staining was minimal in ovaries of reproductively young adult animals, increased in distinct foci in mice of mid-toadvanced reproductive age, and was prominent throughout the ovarian stroma of the oldest animals. Moreover, an infiltration of multinucleated giant cells, peculiar to chronic inflammation, was observed within the ovarian stroma exclusively of reproductively old mice [20].

The female mammalian reproductivity diminishes with age. In uterine aging, the senescence of endometrium contributes to this functional failure. A better understanding of the underlying mechanisms governing the process of uterine aging can hopefully lead to the establishment of new strategies to prolong the reproductive lifespan in humans [21].

\section{References}

1. Ortuño-Sahagún D, Pallàs M, Rojas-Mayorquín AE (2014) Oxidative stress in aging: advances in proteomic approaches. Oxid Med Cell Longev 573208.

2. Phillip JM, Aifuwa I, Walston J, Wirtz D (2015) The Mechanobiology of Aging. Annu Rev Biomed Eng 17:113-141.

3. Walker LC, Herndon JG (2010) Mosaic aging. Med Hypotheses 74(6): 1048-1051. 
4. Wieczorowska-Tobis K (2008) Organ alterations due to aging. Pol Arch Med Wewn 118 Suppl: 63-69.

5. Romano AD, Serviddio G, de Matthaeis A, Bellanti F, Vendemiale G (2010) Oxidative stress and aging. J Nephrol 23 Suppl 15: S29-S36.

6. Holliday R (2004) The multiple and irreversible causes of aging. J Gerontol A Biol Sci Med Sci 59(6): B568-572.

7. Kennedy SR, Loeb LA, Herr AJ (2012) Somatic mutations in aging, cancer and neuro degeneration. Mech Ageing Dev 133(4): 118-126.

8. Le Gall JY, Ardaillou R (2009) The biology of aging. Bull Acad Natl Med 193(2): 365-402; discussion 402-404.

9. Cutler RG, Mattson MP (2006) The adversities of aging. Ageing Res Rev 5(3): 221-238.

10. Yamaguchi O, Otsu K (2012) Role of autophagy in aging. J Cardiovasc Pharmacol 60(3): 242-247.

11. Rezzani R, Stacchiotti A, Rodella LF (2012) Morphological and biochemical studies on aging and autophagy. Ageing Res Rev 11(1): 1031.

12. Lunenfeld B (2006) Endocrinology of the aging male. Minerva Ginecol 58(2): 153-170.

13. Tenover JS (1994) Androgen administration to aging men. Endocrinol Metab Clin North Am 23(4):877-892.

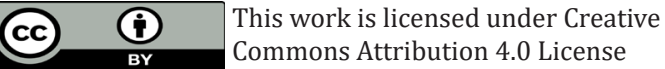

To Submit Your Article Click Here:

Submit Article
DOI: 10.32474/IGWHC.2018.01.000117
14. Kim YC (1999) Testosterone supplementation in the aging male. Int J Impot Res 11(6):343-352.

15. Gunes S, Hekim GN, Arslan MA, Asci R (2016) Effects of aging on the male reproductive system. J Assist Reprod Genet 33(4): 441-454.

16. Amaral S, Ramalho-Santos J (2009) Aging, mitochondria and male reproductive function. Curr Aging Sci 2(3): 165-173.

17. Pierpaoli W (2013) Aging-reversing properties of thyrotropin-releasing hormone. Curr Aging Sci 6(1): 92-98.

18. Shirasuna K, Iwata $\mathrm{H}$ (2017) Effect of aging on the female reproductive function. Contracept Reprod Med 2: 23.

19. Takeuchi M, Matsuzaki K, Nishitani H (2010) Manifestations of the female reproductive organs on MR images: changes induced by various physiologic states. Radiographics 30(4):1147.

20. Briley SM, Jasti S, McCracken JM, Hornick JE, Fegley B, et al. (2016) Reproductive age-associated fibrosis in the stroma of the mammalian ovary. Reproduction 152(3): 245-260.

21. Kong S, Zhang S, Chen Y, Wang W, Wang B, et al. (2012) Determinants of uterine aging: lessons from rodent models. Sci China Life Sci 55(8): 687-693.

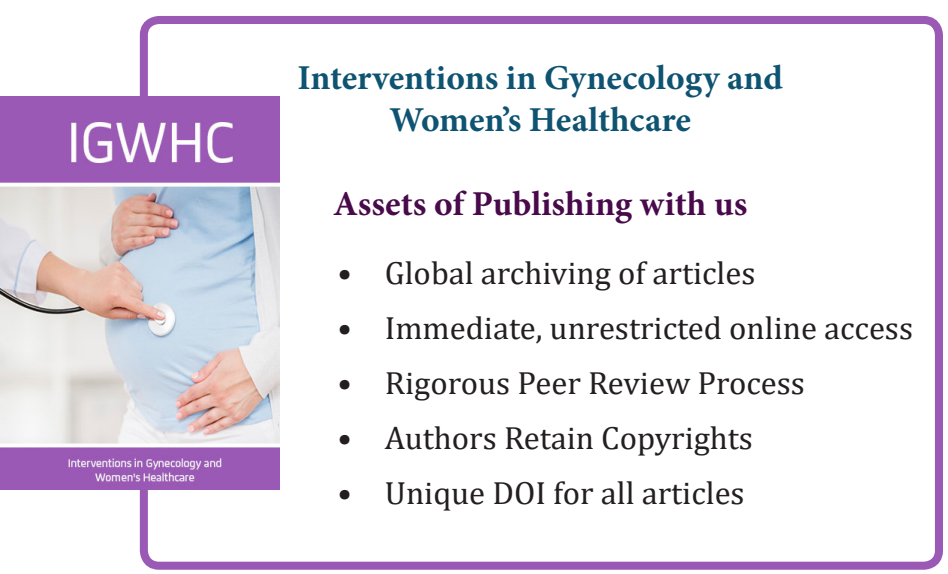

